\title{
ERRATUM
}

F. J. Peñalver • V. Jimenez-Yuste $\cdot$ M. Almagro $\cdot$ A. Alvarez-Larrán $\cdot$ L. Rodríguez M. Casado · L. Gallur • P. Giraldo · R. Hernández $\cdot$ D. Menor • M. J. Rodríguez • D. Caballero $\cdot$ R. González $\cdot$ J. Mayans $\cdot$ I. Millán $\cdot$ J. R. Cabrera $\cdot$ On behalf of the Multi-institutional Retrospective Spanish Study on the use of rituximab in refractory ITP

\section{Rituximab in the management of chronic immune thrombocytopenic purpura: an effective and safe therapeutic alternative in refractory patients}

Published online: 13 May 2006

(C) Springer-Verlag 2006

In the subsection "Factors associated with response", the following incorrect statement was made: "In this group, it was observed that four maintained CR (3 patients that had received three doses and 1 patient that have received one dose)."

The online version of the original article can be found at: http://dx. doi.org/10.1007/s00277-005-0073-1.

\section{F. J. Peñalver}

Fundación Hospital Alcorcón,

Madrid, Spain

V. Jimenez-Yuste

Hospital La Paz,

Madrid, Spain

M. Almagro

Hospital Virgen de las Nieves,

Granada, Spain

\section{A. Alvarez-Larrán}

Hospital Clinic,

Barcelona, Spain

L. Rodríguez

Hospital Germans Trias y Pujol,

Badalona, Spain

M. Casado

Hospital Infanta Cristina,

Badajoz, Spain

L. Gallur

Hospital Vall D' Hebron,

Barcelona, Spain

P. Giraldo

Hospital Miguel Servet,

Zaragoza, Spain

R. Hernández

Hospital de Txagoritxu,

Vitoria, Spain
This sentence should have read: "In this group, it was observed that four maintained CR (three patients that had received three doses and one patient that had received five doses)."

D. Menor

Hospital de Getafe,

Madrid, Spain

M. J. Rodríguez

Hospital Universitario de Canarias,

Tenerife, Spain

D. Caballero

Hospital Clínico de Salamanca,

Salamanca, Spain

R. González

Hospital Sant Joan,

Alicante, Spain

J. Mayans

Hospital Arnau de Vilanova,

Valencia, Spain

I. Millán

Biostatistics Department,

Clínica Puerta de Hierro,

Madrid, Spain

J. R. Cabrera $(\bowtie)$

Hematology Department,

Hospital Universitario Puerta de Hierro,

San Martin de Porres 4,

28035 Madrid, Spain

e-mail: jrcabrera@aehh.org

Tel.: +34-91-3162240

Fax: +34-91-3730535 